Research Paper

\title{
Polymorphism of Nrf2, an Antioxidative Gene, is Associated with Blood Pressure and Cardiovascular Mortality in Hemodialysis Patients
}

\author{
Yasuhiko Shimoyama1 ${ }^{1}$, Yoko Mitsuda², Yoshinari Tsuruta ${ }^{3}$, Nobuyuki Hamajima ${ }^{4}$, Toshimitsu Niwa ${ }^{1,5 \bowtie}$ \\ 1. Department of Advanced Medicine for Uremia, Nagoya University Graduate School of Medicine, 65 Tsurumai-cho, Showa-ku, Nagoya 466-8550, \\ Japan; \\ 2. Department of Preventive Medicine, Nagoya University Graduate School of Medicine, 65 Tsurumai-cho, Showa-ku, Nagoya 466-8550, Japan; \\ 3. Meiyo Clinic, Aichi, Japan; \\ 4. Department of Healthcare Administration, Nagoya University Graduate School of Medicine,65 Tsurumai-cho, Showa-ku, Nagoya 466-8550, \\ Japan; \\ 5. Faculty of Health and Nutrition, Shubun University, 6 Nikko-cho, Ichinomiya, Aichi 491-0938, Japan.
}

$\triangle$ Corresponding author: Prof. Toshimitsu Niwa, MD. Faculty of Health and Nutrition, Shubun University, 6 Nikko-cho, Ichinomiya, Aichi 491-0938, Japan. Tel/Fax: +81-586-43-3114 tniwa@med.nagoya-u.ac.jp.

(c) Ivyspring International Publisher. This is an open-access article distributed under the terms of the Creative Commons License (http://creativecommons.org/ licenses/by-nc-nd/3.0/). Reproduction is permitted for personal, noncommercial use, provided that the article is in whole, unmodified, and properly cited.

Received: 2014.0I.15; Accepted: 2014.04.25; Published: 20I4.05.10

\begin{abstract}
Objective: Nrf2 is a transcription factor that regulates the expression of antioxidant genes. This study aimed to investigate the association of $\mathrm{Nr} 2$ gene single nucleotide polymorphisms (SNPs), rs35652I24 (-653A/G) and rs672/96I (-6I7C/A), with laboratory data and mortality in hemodialysis (HD) patients. Methods: Blood samples were obtained from $216 \mathrm{HD}$ patients (II 9 males and 97 females; 60 diabetics and I56 non-diabetics) with mean age of $60.3 \pm 13.3$ (SD) years, and mean HD duration of $9.10 \pm 8.28$ years. Genotyping was performed using polymerase chain reaction with confronting two-pair primers (PCR-CTPP) assay.

Results: As for rs35652124, diastolic blood pressure (BP) was significantly high in total AA carriers. $\beta 2$-microglobulin was significantly low in male AA carriers. Systolic BP, diastolic BP and albumin were significantly high in female AA carriers. As for 672196I, systolic BP and diastolic BP were significantly high in female AA carriers. Cox proportional hazard analysis adjusted for age, $\mathrm{HD}$ duration, diabetes and $\mathrm{Kt} / \mathrm{V}$ demonstrated that rs35652/24 AA carriers showed higher cardiovascular mortality than $(G G+G A)$ carriers.

Conclusion: Nrf2 SNPs were associated with BP in Japanese HD patients. More notably, rs35652/24 was associated with cardiovascular mortality in these patients.
\end{abstract}

Key words: Nrf2; polymorphism; hemodialysis; blood pressure; cardiovascular mortality.

\section{Introduction}

Nuclear factor-erythroid 2 (NF-E2)-related factor 2 (Nrf2) is a member of the cap'n' collar family of basic leucine zipper transcription factors that regulate the expression of many anti-oxidant pathway genes [1]. Nrf2 is maintained at basal levels in cells by binding to its inhibitor protein, Kelch-like erythroid-cellderived protein with $\mathrm{CNC}$ homology $(\mathrm{ECH})$ associated protein 1 (Keap1) [2,3].
Nrf2 protects many cell types and organ systems from a broad spectrum of toxic insults and disease pathogenesis. For example, Nrf2 protects lung from butylated hydroxytoluene-induced acute respiratory distress syndrome [4], hyperoxic injury [5], and bleomycin-mediated pulmonary fibrosis [6]. Nrf2 increased sensitivity to acetaminophen-induced centrilobular hepatocellular necrosis and hepatotoxicity [7]. 
Nrf2 also contributes to neuro-protection. Activation of the Nrf2-antioxidant response element (ARE) pathway protects neuroblastoma cells from oxidative glutamate toxicity [8] and $\mathrm{H}_{2} \mathrm{O}_{2}$-induced apoptosis [9]. $\mathrm{Nrf} 2$ ameliorates oxidative stress and inflammation in chronic kidney disease [10]. Nrf2 antioxidant functions may be important in vascular diseases [11-13]. Indeed, expression levels of Nrf2-regulated heme oxygenase-1 and glutathione peroxidase-1 play a protective role in atherogenesis [13]. Thus, Nrf2 is called the "multi-organ protector" [14].

Many single nucleotide polymorphisms (SNPs) have been identified in the Nrf2 gene [15-19]. Of special relevance are the rs35652124 (-653A/G) polymorphism and the rs6721961 (-617C/A) polymorphism, which are located in the promotor region of the gene [16]. The rs35652124 and rs6721961 SNPs are predicted to affect Nrf2 myeloid zinc finger 1 (MZF1) and ARE-like promoter binding sites, respectively [15]. These SNPs affect efficient binding of proteins such as Nrf2 to the MZF1 and ARE-like promoter binding sites. Thus, Nrf2 autoregulates its transcription, through these promoter regions. Both SNPs were found to reduce the transcription activity of Nrf2, associated with attenuated binding of Nrf2 to the ARE, resulting in decreased Nrf2-dependent gene transcription. Furthermore, a correlation between individuals carrying the rs6721961CA genotype and increased incidence of acute lung injury, has been reported [15]. Nrf2 gene SNP rs35652124 was associated with nephritis in childhood-onset systemic lupus erythematosus (SLE) [19], and that it might be a risk factor for developing kidney dysfunction in SLE patients. However, there has been no report on Nrf2 SNPs in hemodialysis (HD) patients. This study aimed to investigate the association of Nrf2 gene SNPs, rs35652124 and rs6721961, with various laboratory data, and all-cause and cardiovascular mortality in Japanese HD patients.

\section{Materials and Methods}

\section{Study Subjects}

This study included 216 subjects (119 men and 97 women) taking HD at Meiyo Clinic, Aichi, Japan. The patients included 60 diabetics and 156 non-diabetics, with mean age of $60.3 \pm 13.3$ (SD) years, and mean HD duration of $9.10 \pm 8.28$ years. The allele frequencies of rs35652124 and rs6721961 were compared with 464 subjects taking medical checkup at Nagoya University Hospital. This study was approved by the Ethics Committee of Nagoya University Graduate School of Medicine in 2004.

\section{Laboratory and CACS Measurement}

Blood samples were obtained after 12 hours of fasting. The following biochemical parameters were determined by standard laboratory methods based on Japan Society of Clinical Chemistry (JSCC): total cholesterol, triglyceride, high-density lipoprotein (HDL) cholesterol, low-density lipoprotein (LDL) cholesterol. Coronary artery calcification score (CACS) was measured by using multi-16-detector row computed tomography (Aquilion 16, Toshiba Medical Systems Corporation, Tokyo, Japan) as described previously [20].

\section{Genotyping of Nrf2 Gene SNPs}

Nrf2 SNPs, rs35652124 and rs6721961, were selected from the HapMap database. The genotyping of these SNPs was performed using polymerase chain reaction with confronting two-pair primers (PCR-CTPP) assay [21]. Confronting pairs of primers are as follows:

rs35652124

Forward primer 1:

CTTTTATCTCACTTTACCGCCCGAG

Forward primer 2:

GCAGTCACCCTGAACGCCCT

Reverse primer 1 :

GACACGTGGGAGTTCAGAGGG

Reverse primer 2:

GGGGTTCCCGTTTTTCTCCC

The region containing this polymorphism was amplified by PCR with these primers with the initial denaturation at $95^{\circ} \mathrm{C}$ for $10 \mathrm{~min}$ followed by 30 cycles at $95^{\circ} \mathrm{C}$ for $1 \mathrm{~min}$, at $66^{\circ} \mathrm{C}$ for $1 \mathrm{~min}$, at $72^{\circ} \mathrm{C}$ for $1 \mathrm{~min}$ and additionally at $72^{\circ} \mathrm{C}$ for $5 \mathrm{~min}$. PCR products were visualized on a $2 \%$ agarose gel with ethidium bromide staining. Genotyping was performed as follows; 317, $145 \mathrm{bp}$ for AA genotype, 317, 212, $145 \mathrm{bp}$ for AG genotype, and 317, 212 bp for GG genotype.

rs6721961

Forward primer 1:

CCCTGATTTGGAGGTGCAGAACC

Forward primer 2:

GGGGAGATGTGGACAGCG

Reverse primer 1 :

GCGAACACGAGCTGCCGGA

Reverse primer 2:

CTCCGTTTGCCTTTGACGAC

The region containing this polymorphism was amplified by PCR with these primers with the initial denaturation at $95^{\circ} \mathrm{C}$ for $10 \mathrm{~min}$ followed by 30 cycles at $95^{\circ} \mathrm{C}$ for $1 \mathrm{~min}$, at $58^{\circ} \mathrm{C}$ for $1 \mathrm{~min}$, at $72^{\circ} \mathrm{C}$ for $1 \mathrm{~min}$ and additionally at $72^{\circ} \mathrm{C}$ for $5 \mathrm{~min}$. PCR products were visualized on a $2 \%$ agarose gel with ethidium bromide staining. Genotyping was performed as fol- 
lows; 282, 113 bp for CC genotype, 282, 205, 113 bp for CA genotype, and 282, 205 bp for AA genotype.

\section{Statistical Analysis}

Results are expressed as mean \pm SD. Hardy-Weinberg equilibrium testing was performed by using the $X^{2}$ test. Student's $t$ test and multivariate analysis adjusted for age and duration on HD were performed in comparison of the mean values between the different genotype groups.

The associations of the SNPs with all-cause mortality and cardiovascular mortality were evaluated. In this study, cardiovascular disease was defined as apparent heart disease, cerebrovascular disease and peripheral artery disease as a primary cause of death. Kaplan-Meier method and Cox proportional hazard analysis were used to examine the impact of these SNPs on survival after adjusting for age and HD duration. Significance was defined as a $\mathrm{p}$ value of $<0.05$. All analysis was done by using SPSS statistics 20 (SPSS Japan Inc., Tokyo, Japan).

\section{Results}

\section{Incidence of Nrf2 Gene SNPs}

Table 1 shows general characteristics of HD patients. Table 2 shows genotype frequencies of $N r f 2$ gene. A strong linkage was observed between these two SNPs $\left(\mathrm{D}^{\prime}=0.983, \mathrm{r}^{2}=0.507\right)$. The incidences of genotypes in Nrf2 gene in healthy subjects and HD patients are shown in Table 3. As for HD patients, the allele frequencies of the rs 35652124 polymorphism of the Nrf2 gene were 0.574 for the G allele and 0.426 for the A allele, which was not significantly different from healthy subjects. The allele frequencies of the rs6721961 polymorphism of the Nrf2 gene were 0.720 for the C allele and 0.280 for the A allele, which was not significantly different from healthy subjects. The genotype distributions for the rs35652124 and rs6721961 were in Hardy-Weinberg equilibrium ( $p=0.61$ and 0.46 , respectively).

Table I. General characteristics of subjects.

\begin{tabular}{|c|c|c|c|c|}
\hline & & Total & Male & Female \\
\hline $\mathrm{n}$ & & 216 & 119 & 97 \\
\hline Age & (year) & $60.3 \pm 13.3$ & $58.7 \pm 13.3$ & $62.4 \pm 13.1$ \\
\hline HD-duration & (year) & $9.10 \pm 8.28$ & $8.75 \pm 8.53$ & $9.54 \pm 7.99$ \\
\hline Diabetes & (yes / no) & $60 / 156$ & $43 / 76$ & $17 / 80$ \\
\hline Systolic BP & $(\mathrm{mmHg})$ & $143.2 \pm 23.9$ & $148.2 \pm 21.5$ & $136.3 \pm 25.5$ \\
\hline Diastolic BP & $(\mathrm{mmHg})$ & $75.3 \pm 13.5$ & $76.8 \pm 13.3$ & $73.3 \pm 13.5$ \\
\hline Red blood cell & $\left(\times 10^{6} / \mu \mathrm{l}\right)$ & $3.28 \pm 0.37$ & $3.34 \pm 0.39$ & $3.21 \pm 0.34$ \\
\hline Hematocrit & $(\%)$ & $31.7 \pm 3.1$ & $32.2 \pm 3.2$ & $31.0 \pm 2.8$ \\
\hline White blood cell & $\left(\times 10^{3} / \mu \mathrm{l}\right)$ & $5.87 \pm 1.71$ & $6.12 \pm 1.79$ & $5.55 \pm 1.56$ \\
\hline Platelet & $\left(\times 10^{3} / \mu \mathrm{l}\right)$ & $183 \pm 55$ & $183 \pm 56$ & $182 \pm 55$ \\
\hline $\mathrm{LDH}$ & (IU/l) & $349 \pm 70$ & $344 \pm 65$ & $355 \pm 76$ \\
\hline ALP & (IU/1) & $166 \pm 76$ & $149 \pm 57$ & $187 \pm 90$ \\
\hline Total protein & $(\mathrm{g} / \mathrm{l})$ & $70.0 \pm 4.6$ & $70.4 \pm 4.7$ & $69.4 \pm 4.4$ \\
\hline Albumin & $(\mathrm{g} / \mathrm{l})$ & $41.3 \pm 3.6$ & $41.9 \pm 3.5$ & $40.5 \pm 3.5$ \\
\hline Total cholesterol & $(\mathrm{mmol} / \mathrm{l})$ & $4.40 \pm 0.93$ & $42.1 \pm 0.91$ & $4.63 \pm 0.90$ \\
\hline Triglyceride & $(\mathrm{mmol} / \mathrm{l})$ & $1.55 \pm 1.20$ & $1.67 \pm 1.49$ & $1.41 \pm 0.66$ \\
\hline HDL cholesterol & $(\mathrm{mmol} / \mathrm{l})$ & $1.14 \pm 0.33$ & $1.05 \pm 0.28$ & $1.24 \pm 0.35$ \\
\hline LDL cholesterol & $(\mathrm{mmol} / \mathrm{l})$ & $2.58 \pm 0.76$ & $2.44 \pm 0.68$ & $2.74 \pm 0.81$ \\
\hline Uric acid & $(\mu \mathrm{mol} / \mathrm{l})$ & $490 \pm 79$ & $499 \pm 86$ & $479 \pm 68$ \\
\hline $\mathrm{HbA1c}$ & $(\%)$ & $7.36 \pm 1.40$ & $7.40 \pm 1.51$ & $7.29 \pm 1.14$ \\
\hline Calcium (Ca) & $(\mathrm{mmol} / \mathrm{l})$ & $2.14 \pm 0.23$ & $2.14 \pm 0.24$ & $2.14 \pm 0.22$ \\
\hline Phosphorus (P) & $(\mathrm{mmol} / \mathrm{l})$ & $1.91 \pm 0.37$ & $1.94 \pm 0.43$ & $1.88 \pm 0.29$ \\
\hline $\mathrm{Ca} \times \mathrm{P}$ & $(\mathrm{mmol} / \mathrm{l})^{2}$ & $4.09 \pm 0.97$ & $4.16 \pm 1.08$ & $4.02 \pm 0.82$ \\
\hline Homocysteine & $(\mu \mathrm{mol} / \mathrm{l})$ & $37.0 \pm 22.7$ & $38.9 \pm 22.8$ & $34.6 \pm 22.5$ \\
\hline Malondialdehyde & $(\mathrm{mg} / \mathrm{l})$ & $1.68 \pm 0.29$ & $1.68 \pm 0.31$ & $1.69 \pm 0.26$ \\
\hline Lipid peroxides & $(\mu \mathrm{mol} / \mathrm{l})$ & $1.23 \pm 0.86$ & $1.17 \pm 0.69$ & $1.30 \pm 1.03$ \\
\hline Carboxymethyl lysine & $(\mathrm{mg} / \mathrm{l})$ & $9.85 \pm 2.05$ & $9.74 \pm 2.13$ & $9.99 \pm 1.94$ \\
\hline C-reactive protein (CRP) & $(\mathrm{nmol} / \mathrm{l})$ & $21.9 \pm 54.6$ & $19.8 \pm 43.1$ & $24.5 \pm 66.2$ \\
\hline$\beta 2$-microglobulin & $(\mathrm{mg} / \mathrm{l})$ & $32.8 \pm 7.3$ & $32.4 \pm 7.8$ & $33.3 \pm 6.8$ \\
\hline Pentosidine & $(\mathrm{mg} / \mathrm{l})$ & $0.446 \pm 0.149$ & $0.451 \pm 0.164$ & $0.440 \pm 0.129$ \\
\hline Indoxyl sulfate & $(\mathrm{mg} / \mathrm{l})$ & $35.4 \pm 14.7$ & $36.5 \pm 14.5$ & $34.0 \pm 14.9$ \\
\hline High-sensitive PTH & (ng/l) & $19233 \pm 15787$ & $21496 \pm 17857$ & $16510 \pm 12464$ \\
\hline Troponin $\mathrm{T}$ & $(\mu \mathrm{g} / 1)$ & $0.031 \pm 0.056$ & $0.038 \pm 0.066$ & $0.023 \pm 0.038$ \\
\hline CACS & $\left(\mathrm{mm}^{3}\right)$ & $1283 \pm 1914$ & $1432 \pm 2061$ & $1099 \pm 1710$ \\
\hline $\mathrm{Kt} / \mathrm{V}$ & & $1.34 \pm 0.24$ & $1.23 \pm 0.20$ & $1.49 \pm 0.19$ \\
\hline
\end{tabular}

Data are expressed as mean $\pm \mathrm{SD}$. 
Table 2. Genotype frequencies of Nrf2 gene.

\begin{tabular}{llllll}
\hline \multicolumn{5}{c}{ rs6721961 } \\
\hline \multirow{2}{*}{ rs35652124 } & & CC & CA & AA & Total \\
& GG & 73 & 0 & 0 & 73 \\
& AG & 31 & 70 & 1 & 102 \\
& AA & 10 & 13 & 18 & 41 \\
& Total & 114 & 83 & 19 & 216 \\
\hline
\end{tabular}

Table 3. Comparison between HD patients and healthy controls in each genotype of $\mathrm{Nrf2}$ gene.

\begin{tabular}{lllll}
\hline & & Healthy subjects & HD patients & $\mathrm{p}$ value \\
\hline rs35652124 & GG & 162 & 73 & \\
& GA & 204 & 102 & \\
& AA & 98 & 41 & \\
A allele percentage & & $43.1 \%$ & $42.6 \%$ & 0.694 \\
rs7895833 & CC & 256 & 114 & \\
& CA & 161 & 83 & \\
& AA & 47 & 19 & \\
A allele percentage & & $27.5 \%$ & $28.0 \%$ & 0.608 \\
\hline
\end{tabular}

\section{Association of Nrf2 Gene SNPs with Various Variables}

Table 4 shows association of SNP rs35652124 with variables in HD patients. In total patients, diastolic blood pressure (BP) and albumin were significantly high in AA carriers. Troponin T was signifi- cantly low in AA carriers. In male patients, $\beta 2$-microglobulin, CACS and troponin T were significantly low in AA carriers. In female patients, systolic $\mathrm{BP}$, diastolic BP, and albumin were significantly high in AA carriers.

We performed multivariate analysis adjusted for age, duration on HD, diabetes (yes or no) and Kt/V. In total patients, diastolic BP was significantly high in AA carriers. In male patients, $\beta 2$-microglobulin was significantly low in AA carriers. In female patients, systolic BP, diastolic BP, and albumin were significantly high in AA carriers.

Table 4 shows association of SNP rs6721961 with variables in HD patients. In total patients, homocysteine and troponin $\mathrm{T}$ were significantly low in AA carriers. In male patients, HD duration, homocysteine, CACS, and troponin T were significantly low in AA carriers. In female patients, systolic BP, diastolic BP, and albumin were significantly high in AA carriers. Platelet was significantly low in AA carriers.

We performed multivariate analysis adjusted for age, duration on $\mathrm{HD}$, diabetes, and $\mathrm{Kt} / \mathrm{V}$. In female patients, systolic and diastolic BP levels were significantly high in AA carriers. Platelet was significantly low in AA carriers. In male patients, however, there were no significant differences in the variables between the genotypes.

Table 4. Variables according to polymorphism in Nrf2 gene.

\begin{tabular}{|c|c|c|c|c|c|c|c|c|c|}
\hline & & rs35652124 & & & & rs6721961 & & & \\
\hline & & GG+GA & AA & p value 1 & $\mathrm{p}$ value ${ }^{2}$ & $\mathrm{CC}+\mathrm{CA}$ & $\mathrm{AA}$ & $\mathrm{p}$ value $^{1}$ & $\mathrm{p}$ value ${ }^{2}$ \\
\hline \multicolumn{10}{|l|}{ Total } \\
\hline $\mathrm{n}$ & & 175 & 41 & & & 197 & 19 & & \\
\hline Diastolic BP & $(\mathrm{mmHg})$ & $74.2 \pm 13.1$ & $79.9 \pm 14.2$ & 0.024 & 0.047 & & & & \\
\hline Albumin & $(\mathrm{g} / 1)$ & $41.0 \pm 3.5$ & $42.4 \pm 3.9$ & 0.018 & ns & & & & \\
\hline Homocysteine & $(\mu \mathrm{mol} / 1)$ & & & & & $37.62 \pm 23.61$ & $30.03 \pm 7.14$ & 0.002 & ns \\
\hline Troponin $\mathrm{T}$ & $(\mu \mathrm{g} / \mathrm{l})$ & $0.035 \pm 0.060$ & $0.016 \pm 0.032$ & 0.006 & ns & $0.033 \pm 0.058$ & $0.014 \pm 0.018$ & 0.001 & ns \\
\hline
\end{tabular}

Male

$\mathrm{n}$

HD-duration

Homocysteine

32-microglobulin

CACS

Troponin T

Female

$\mathrm{n}$

Systolic BP

Diastolic BP

Platelet

Albumin
99

(year)

( $\mu \mathrm{mol} / \mathrm{l})$

(mg/1)

$\left(\mathrm{mm}^{3}\right)$

( $\mu \mathrm{g} / 1)$

\section{$33.3 \pm 7.6$}

$1605 \pm 2165$

$0.042 \pm 0.070$
20

$\begin{array}{ll}27.9 \pm 7.8 & 0.007 \\ 579 \pm 1186 & 0.003 \\ 0.019 \pm 0.036 & 0.030\end{array}$

108

$9.25 \pm 8.73$

$39.89 \pm 23.67$

0.034

ns

ns

$1533 \pm 2130$

$0.041 \pm 0.069$

11

$3.83 \pm 3.56$

$28.94 \pm 6.73$

$444 \pm 630$

0.001 ns

${ }^{1}$ Unadjusted analysis (Student's t-test or Welch's t-test)

${ }^{2}$ Multivariate analysis adjusted for age, HD-duration, diabetes (yes or no) and Kt/V

ns: not significant 


\section{Survival Analysis of Nrf2 Gene SNPs}

Figure 1 shows estimates of survival by the recessive model of rs35652124 genotype (A: all-cause, B: cardiovascular). The cumulative all-cause mortality values of genotype (GG+GA) and genotype AA were $32.7 \%$ and $36.8 \%$, respectively. The cumulative cardiovascular mortality values of genotype (GG+GA) and genotype AA were $13.5 \%$ and $28.9 \%$, respectively. In this model, cardiovascular mortality was significantly high in AA carriers of rs35652124 compared with $(G G+G A)$ genotype $(p=0.032)$. Cox proportional hazard analysis adjusted for age and HD duration demonstrated that AA carriers showed significantly high cardiovascular mortality compared with $(G G+G A)$ carriers (odds ratio $=2.834, p=0.006$ ). This trend was more prominent in female patients. The cumulative cardiovascular mortality values of genotype (GG+GA) and genotype AA in female were $13.2 \%$ and $42.1 \%(p=0.007)$, respectively. Cox proportional hazard analysis adjusted for age and HD duration demonstrated that the odds ratio of AA carriers/(GG+GA) carriers was significantly high (odds ratio $=5.856, \mathrm{p}=0.001)$.

Figure 2 shows estimates of survival by the recessive model of rs6721961 genotype (A: all-cause, B: cardiovascular). There was no significant difference in the mortality between the genotype groups.
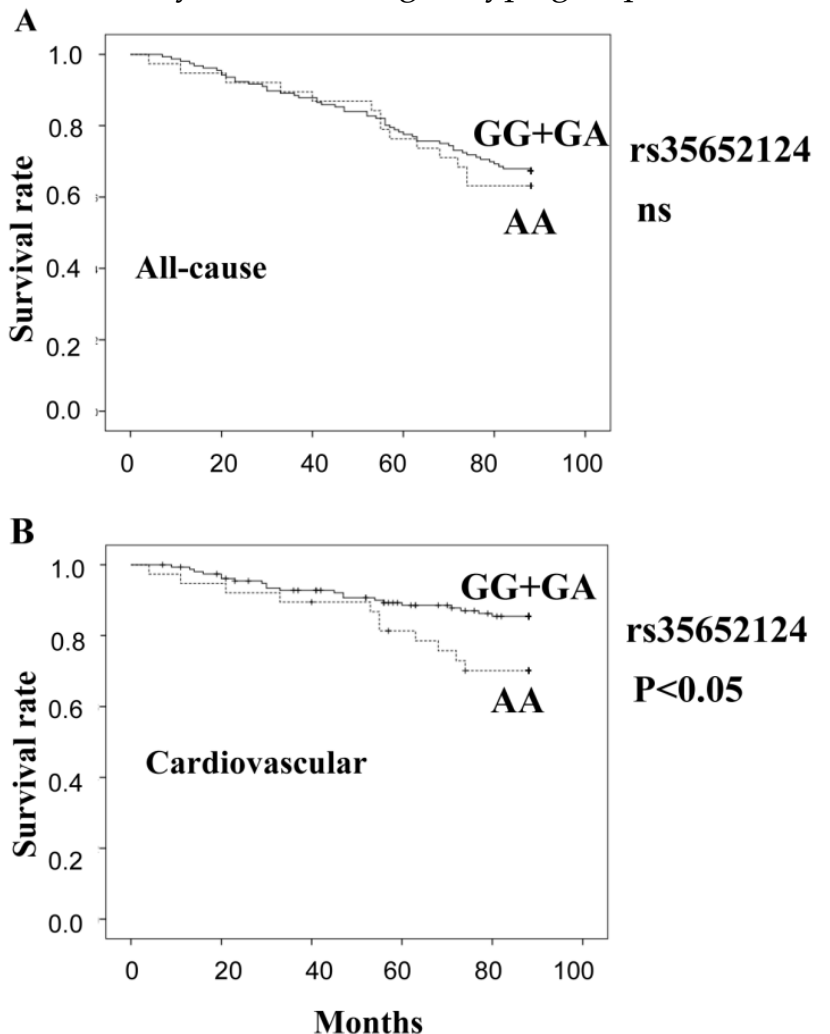

Figure I. Survival curves of all-cause mortality (A) and cardiovascular mortality (B), calculated by the Kaplan Meier method, based on the SNP rs35652 I24. (GG+GA) genotype: solid line, AA genotype: broken line. ns: not significant.
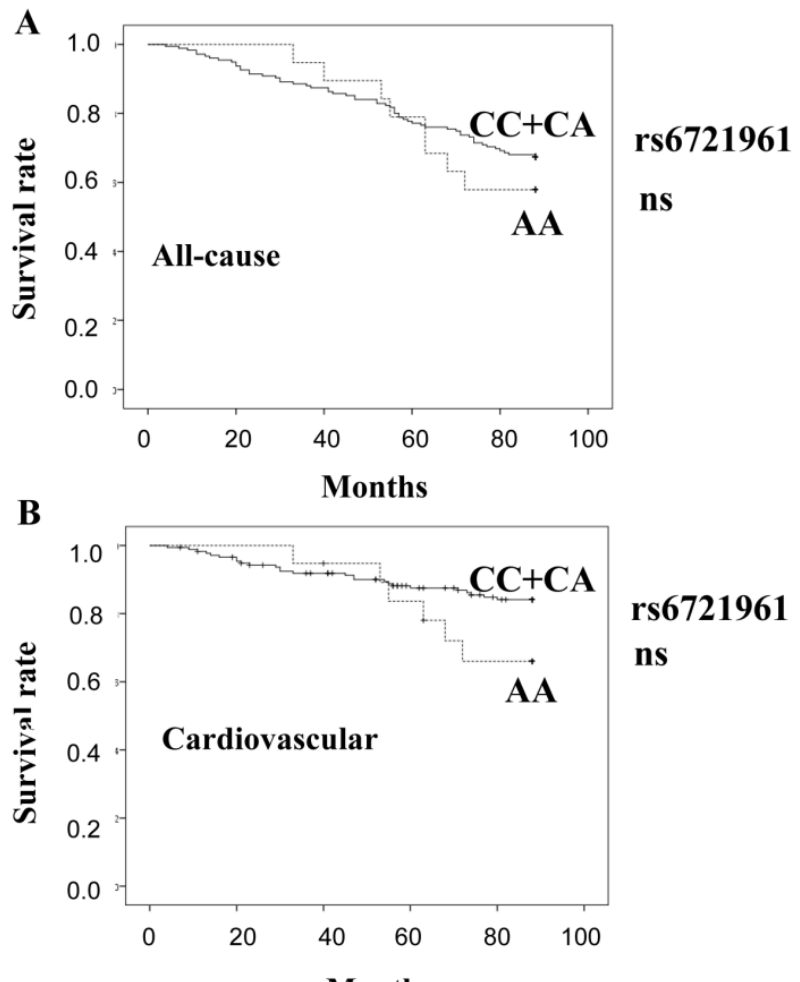

Figure 2. Survival curves of all-cause mortality (A) and cardiovascular mortality (B), calculated by the Kaplan Meier method, based on the SNP rs672196I. (CC+CA) genotype: solid line, AA genotype: broken line. ns: not significant.

\section{Discussion}

The novel findings of the present study are: 1) systolic and diastolic BP levels were significantly high in rs35652124 AA carriers and rs6721961 AA carriers in HD patients, especially in female patients, and 2) cardiovascular mortality was significantly high in rs35652124 AA carriers in HD patients, especially in female patients. Thus, Nrf2 gene SNPs, rs35652124 and rs6721961, were associated with BP in Japanese HD patients. More notably, SNP rs35652124 was associated with cardiovascular mortality in these patients.

The present study revealed that cardiovascular mortality as well as systolic and diastolic BP was significantly high in A allele carriers of rs35652124. This tendency was more prominent in female patients. We consider that high cardiovascular mortality in the A allele carriers of rs35652124 might be due to high BP, because hypertension causes a variety of cardiovascular diseases such as atherosclerosis, myocardial infarction and cerebral infarction.

A recent study demonstrated association between Nrf2 polymorphism and hemodynamic parameters. Polymorphisms (rs35652124 and rs6721961) within the Nrf2 promoter were associated with impaired forearm vasodilator responses in an endothe- 
lial-independent manner, suggesting an important role of Nrf2 in the regulation of vascular function in humans [16]. These vascular responses have been shown to correlate with vasodilator responses in coronary arteries, and predict the risk of developing cardiovascular disease [22]. The present study suggests that different transcription activity due to Nrf2 gene polymorphisms might affect BP by modulating protection against cellular oxidative stress. $\mathrm{Wu}$ et al. demonstrated that oxidative stress is associated with elevated BP [23]. Imbalance between production and scavenging of superoxide anion results in hypertension by the inactivation of nitric oxide, and the increased oxidative stress from the resultant peroxynitrite promotes inflammatory processes such as atherosclerosis.

Marzec et al. reported that Nrf2 activity was low in G allele carriers of rs35652124 and A allele carriers of rs6721961 [15]. We hypothesized that cardiovascular mortality might be higher in $G$ allele carriers of rs35652124 and/or A allele carriers of rs6721961. However, contrary to our hypothesis, the present study revealed that cardiovascular mortality was lower in G allele carriers of rs35652124, but not in A allele carriers of rs6721961. Thus, low activity of Nrf2 as shown in G allele carriers of rs35652124 seems to be associated with low cardiovascular mortality.

Sussan et al. reported that Nrf2 knockout mice surprisingly have a decreased susceptibility to ApoE-mediated atherosclerotic plaque formation, and suggested that the pro-atherogenic effect of Nrf2 may be mediated via positive regulation of the scavenger receptor (CD36) [24]. Nrf2-mediated CD36 expression is a major pathway by which modified LDL becomes incorporated into atheroma. In Nrf2 deficiency, even in greater oxidative stress, atherosclerosis is reduced. Barajas et al. also found that Nrf2 knockout mice unexpectedly exhibited reduction in the degree of aortic atherosclerosis compared with wild-type controls, and therefore concluded that Nrf2 expression promotes atherosclerotic lesion formation, by a combination of systemic metabolic and local vascular effects [25]. Decreased atherosclerosis in the Nrf2 knockout mice correlated with lower plasma total cholesterol levels. Thus, Nrf2 is pro-atherogenic, despite its antioxidative function. These findings might explain why low activity of Nrf2 as shown in G allele carriers of rs35652124 was associated with low cardiovascular mortality in our patients.

In conclusion, Nrf2 SNPs, rs35652124 and rs6721961, were associated with BP in Japanese HD patients. More importantly, Nrf2 SNP rs35652124 was associated with cardiovascular mortality in these patients. Further study on Nrf2 SNPs with a larger group of HD patients might be necessary to confirm our findings.

\section{Competing Interests}

The authors have declared that no competing interest exists.

\section{References}

1. Kensle TW, Wakabayashi N, Biswal S. Cell survival responses to environmental stresses via the Keap1-Nrf2-ARE pathway. Annu Rev Pharmacol Toxicol. 2007; 47: 89-106.

2. Dhakshinamoothy S, Jaiswal AK. Functional characterization and role of INrf2 in antioxidant response element-mediated expression and antioxidant induction of NAD(P)H:quinone oxidoreductase1 gene. Oncogene. 2001; 20: 3906-17.

3. Itoh K, Wakabayashi N, Katoh Y, et al. Keap1 represses nuclear activation of antioxidant responsive elements by Nrf2 through binding to the amino-terminal Neh2 domain. Genes Dev. 1999; 13: 76-86.

4. Chan K, Kan YW. Nrf2 is essential for protection against acute pulmonary in jury in mice. Proc Natl Acad Sci USA. 1999; 96: 12731-6.

5. Cho HY, Jedlicka AE, Reddy SP, et al. Role of NRF2 in protection against hyperoxic lung injury in mice. Am J Respir Cell Mol Biol. 2002; 26: 175-82.

6. Cho HY, Reddy SP, Yamamoto M, et al. The transcription factor NRF2 protects against pulmonary fibrosis. FASEB J. 2004; 18: 1258-60.

7. Enomoto A, Itoh K, Nagayoshi E, et al. High sensitivity of Nrf2 knockout mice to acetaminophen hepatotoxicity of Nrf2 knockout mice to acetaminophen hepatotoxicity associated with decreased expression of ARE-regulated drug metabolizing enzymes and antioxidant genes. Taxicol Sci. 2001; 59: 169-77.

8. Murphy TH, De Long MJ, Coyle JT. Enhanced NAD(P)H:quinone reductase activity prevents glutamate toxicity produced by oxidative stress. J Neurochem. 1991; 56: 990-5

9. Li J, Lee JM, Johnson JA. Microarray analysis reveals an antioxidant responsive element-driven gene set involved in conferring protection from an oxidative stress-induced apoptosis in IMR-32 cells. J Biol Chem. 2002; 277: 388-94.

10. Ruiz S, Pergola PE, Zager RA, et al. Targeting the transcription factor Nrf2 to ameliorate oxidative stress and inflammation in chronic kidney disease. Kidney Int. 2013; 83: 1029-41.

11. Levonen $\mathrm{AL}$, Inkala $\mathrm{M}$, Heikura $\mathrm{T}$, et al. Nrf2 gene transfer induces antioxidant enzymes and suppresses smooth muscle cell growth in vitro and reduces oxidative stress in rabbit aorta in vivo. Arterioscler Thromb Vasc Biol. 2007; 27: 741-7.

12. Jyrkkanen HK, Kansanen E, Inkala M, et al. Nrf2 regulates antioxidant gene expression evoked by oxidized phospholipids in endothelial cells and murine arteries in vivo. Circ Res. 2008; 103: e1-9.

13. Yet SF, Layne MD, Liu X, et al. Absence of heme oxygenase-1 exacerbates atherosclerotic lesion formation and vascular remodeling. FASEB J. 2003; 17: 1759-61.

14. Lee JM, Li J, Johnson DA, Stein TD, et al. Nrf2, a multi-organ protector? FASEB J. 2005; 19: 1061-4.

15. 15 Marzec JM, Christie JD, Reddy SP, et al. Functional polymorphisms in the transcription factor NRF2 in humans increase the risk of acute lung injury. FASEB J. 2007; 21: $2237-46$

16. Marczak ED, Marzec J, Zeldin DC, et al. Polymorphisms in the transcription factor NRF2 and forearm vasodilator responses in humans. Pharmacogenet Genomics. 2012; 22: 620-8.

17. Yamamoto $\mathrm{T}$, Yoh $\mathrm{K}$, Kobayashi A, et al. Identification of polymorphisms in the promoter region of the human NRF2 gene. Biochem Biophys Res Commun. 2004; 321: 72-9.

18. Siedlinski M, Postma DS, Boer JM, et al. Level and course of FEV1 in relation to polymorphisms in NFE2L2 and KEAP1 in the general population. Respir Res. 2009; 10: 73

19. Córdova EJ, Velázquez-Cruz R, Centeno F, et al. The NRF2 gene variant, -653G/A, is associated with nephritis in childhood-onset systemic lupus erythematosus. Lupus. 2010; 19: 1237-42

20. Taki K, Takayama F, Tsuruta Y, et al. Oxidative stress, advanced glycation end product and coronary artery calcification in hemodialysis patients. Kidney Int. 2006; 70: 218-24.

21. Hamajima N, Saito T, Matsuo K, et al. Polymerase chain reaction with confronting two-pair primers for polymorphism genotyping. Jpn J Cancer Res. 2000; 91: 865-8.

22. Heitzer T, Schlinzig T, Krohn K, et al. Endothelial dysfunction, oxidative stress, and risk of cardiovascular events in patients with coronary artery disease. Circulation. 2001; 104: 2673-8.

23. Wu L, Noyan Ashraf MH, Facci M, et al. Dietary approach to attenuate oxidative stress, hypertension, and inflammation in the cardiovascular system. Proc Natl Acad Sci USA. 2004; 101: 7094-9.

24. Sussan TE, Jun J, Thimmulappa R, et al. Disruption of Nrf2, a key inducer of antioxidant defenses, attenuates ApoE-mediated atherosclerosis in mice. PLoS One. 2008; 3 : e3791.

25. Barajas B, Che N, Yin F, et al. NF-E2-related factor 2 promotes atherosclerosis by effects on plasma lipoproteins and cholesterol transport that overshadow antioxidant protection. Arterioscler Thromb Vasc Biol. 2011; 31: 58-66. 